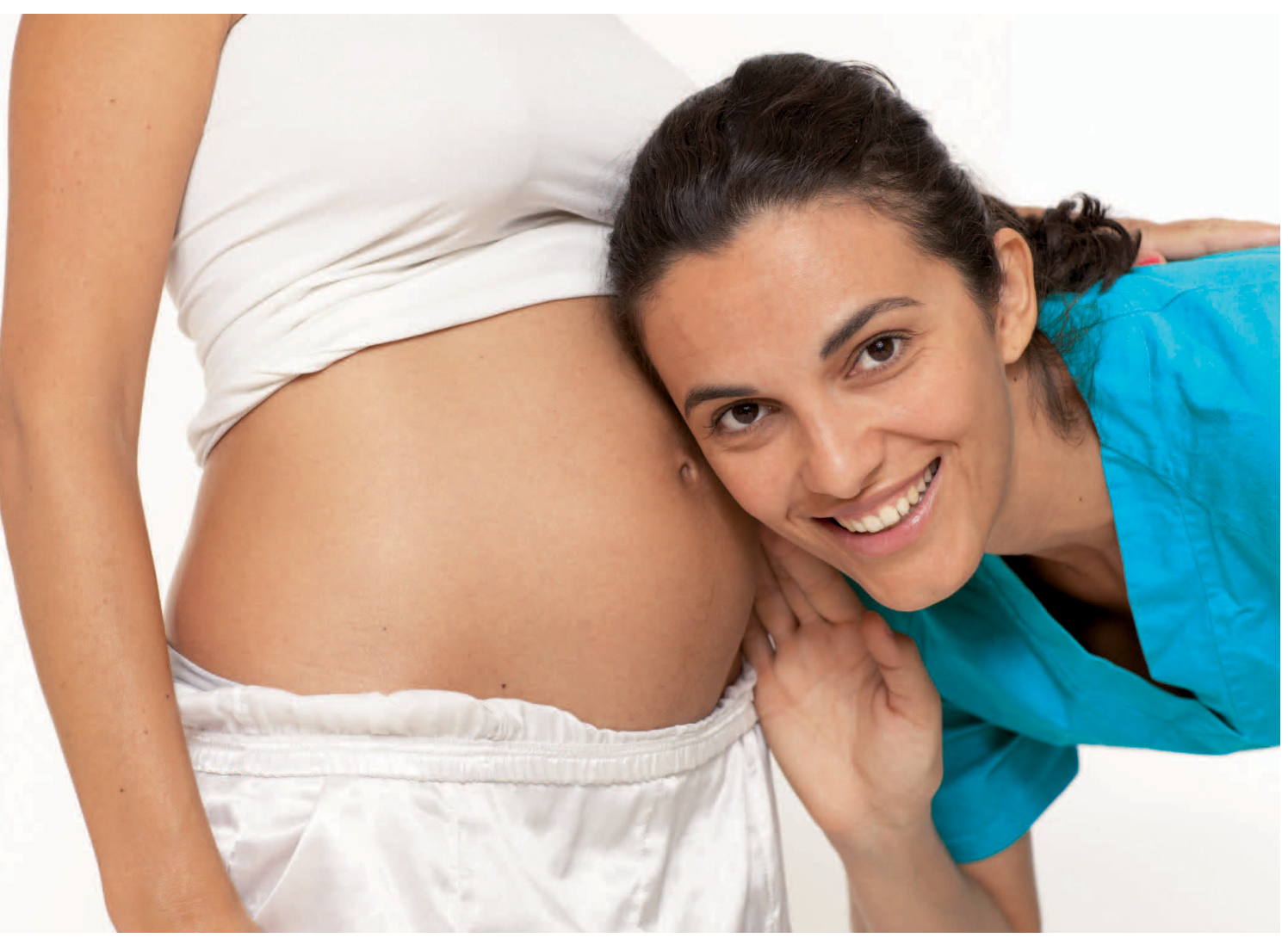

\title{
Schwanger - na und?
}

SCHWANGER AM ARBEITSPLATZ Früher war es absolute Routine: schwangere Mitarbeiterinnen auf der Intensivstation oder in der Anästhesie. Doch das änderte sich mit immer mehr gesetzlichen Auflagen, die diese gängige Praxis zunehmend erschweren. Heute ist schnell ein Beschäftigungsverbot ausgesprochen. Dennoch gibt es Intensiv- und Anästhesie-Teams, die eine Mitarbeit während der Schwangerschaft ermöglichen und die sich über eine Rückkehr der Mütter ins Berufsleben freuen.

Monika Hiltensperger

Katja Mülder steht in blauer Funktionskleidung hinter der Eingangstür der Kinderintensivstation der Kölner Uniklinik. Sie ist als Teamleitung für etwa 60 Mitarbeiter zuständig. Neben der Kinderintensiv zählt auch die Zentrale Notaufnahme zu ihrem Team. Mülder flitzt mit schnellen Schritten in ihr Büro. Erst als sie sich umdreht, zeigt sich ihre Schwangerschaft mit einem beachtlichen Bauchumfang und einem freudestrahlenden Gesicht. Inzwischen ist sie in der 30. Schwangerschaftswoche. Sie sagt, es gehe ihr gut. Auf die Frage, wie sich Nichtschwange- re das Arbeiten mit dem üppigen Bauch und der ungewohnten Hormonkonstellation vorstellen könnten, lacht sie: „Es ist schon mühsamer und fühlt sich für mich an manchen Tagen etwa so an wie früher, wenn ich die halbe Nacht durchgefeiert habe und früh morgens wieder arbeiten musste." Die scheinbar durchzechten Nächte sind Katja Mülder nicht anzusehen, sie sieht beinahe so munter aus, als hätte sie gerade einen Urlaub hinter sich. Doch den hat sie noch vor sich - wohlweislich hat sie ihn bis zum Schluss aufgehoben. Auch ihren schwangeren Mitarbeiterinnen legt sie das immer wieder nahe: „Je fortgeschrittener die Schwangerschaft ist, umso anstrengender wird ein Vollzeitjob. So ist der zu erwartende Urlaub vor dem Mutterschutz besonders erfreulich." Alle Überstunden baut sie in Absprache mit den schwangeren Frauen möglichst bald ab, sodass zu Beginn des Mutterschutzes das Arbeitszeitkonto ausgeglichen ist. Der Mutterschutz beginnt sechs Wochen vor und endet acht Wochen nach der Entbindung.

\section{Schwangere im Klinikalltag}

Die Uniklinik Köln praktiziert erfolgreich die Integration von Schwangeren in den 


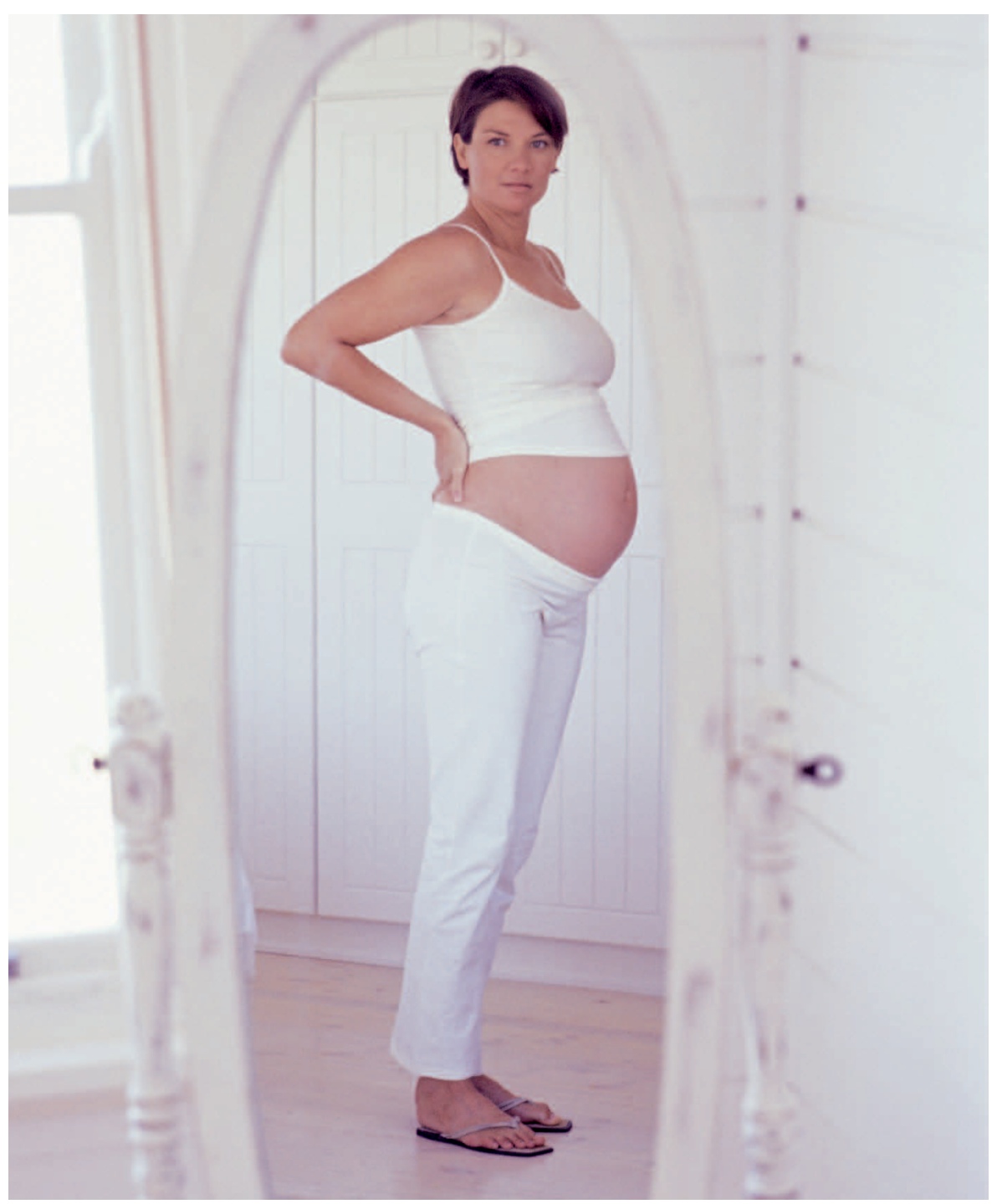

Anästhesie- oder Intensivalltag, die das auch ausdrücklich wünschen. In der Erwachsenen-Intensivpflege arbeiten viele ganz normal weiter, sie bekommen allerdings nicht die „schweren Fälle“. Für alle pflegenden Schwangeren gilt: Sie versorgen keine infektiösen und keine intubierten Patienten mehr, auch adipöse übernehmen die Kollegen. Schwangere Fachkräfte in der Anästhesie arbeiten nicht mehr in der Nähe von Inhalationsnarkosen und Röntgenstrahlen, sind nicht im Aufwachraum und fahren keine Transporte. Stattdessen assistieren sie bei regionalen oder örtlichen Betäubungen und helfen dort, wo Lachgasnarkosen selten sind wie in der Herzchirurgie oder der Gynäkologie.

Vorausgesetzt, es liegt kein Beschäftigungsverbot vor, stellt es Mülder den
Immerhin leistete Mülder den organisatorischen Spagat beim Umschreiben von Dienstplänen, nachdem ihr der Dominoeffekt in den letzten zwei Jahren neun Schwangere beschert hat. Die Mehrheit ihrer schwangeren Mitarbeiterinnen möchte lieber aus der Pflege raus und zieht eine Bürotätigkeit vor. Hier leisten sie wertvolle Dienste. Mit Mitarbeiterinnen, die lieber in der Pflege weiterarbeiten, spricht Mülder ausführlich. Sie weist sie verstärkt auf ihre Möglichkeit hin, sich selbst und ihr werdendes Kind mittels Hygienemaßnahmen wie Schutzkleidung und Handschuhen vor Infektionen zu schützen - was in einer Abteilung mit kleinen Kindern besonders in puncto Zytomegalie und Toxoplasmose wichtig ist. Immerhin haben schwangere Pflegekräfte bessere Möglichkeiten sich zu schützen als andere Berufsgruppen wie beispielsweise Verkäuferinnen. Pflegeprofis kennen die Übertragungswege von Infektionen und sollten dieses Wissen unbedingt nutzen. Im Gegensatz zur häuslichen Umgebung mit Hort- oder Kindergartenkindern stehen ihnen im Krankenhaus auch die notwendigen Schutzmittel zur Verfügung. Mülder appelliert an die Verantwortung der Schwangeren: Sie sollen sich selbst um ihre Sicherheit kümmern, nicht darauf warten, dass andere das tun, und bei Bedarf Kollegen zur Hilfe holen.

\section{Transparenz ist der beste Schutz}

Im Intranet der Kölner Klinik finden sich alle wichtigen Informationen für Schwangere gebündelt: das Mutterschutzgesetz (MuSchG), die Mutterschutzrichtlinienverordnung (MuSchRiV), die Strahlenschutzverordnung (StrlSchV), die Röntgenverordnung (RöV) und die technischen Regeln für Gefahrstoffe (TRGS). Sobald eine Kollegin weiß, dass sie schwanger ist, legt sie ihren Mutterpass vor. Dann beginnt der Ablauf: Die Personalverwaltung braucht die Meldung, damit sie die Schichtzulage weiter vergüten kann, obwohl sich die tatsächlich geleistete Arbeitszeit auf zwei Schichten zwischen 6:30 Uhr und $20 \mathrm{Uhr}$ reduziert. Die Teamleitung schreibt den Dienstplan um, nimmt die Kollegin sofort aus der Nachtschicht und dem normalen Spätdienst (über 20 Uhr hinaus). Sie fordert Mitarbeiter aus dem hausinternen Intensiv-Pool an. Der Arbeitgeber meldet 
die Schwangerschaft der Mitarbeiterin beim Gewerbeaufsichtsamt. Von der Teamleitung bekommt die werdende Mutter einen Gefährdungsbeurteilungsbogen in die Hand, diesen füllen sie gemeinsam aus. Mit diesem Papier geht die werdende Mutter zum Betriebsarzt. Der Betriebsarzt erklärt ihr, worauf sie während der Schwangerschaft bei ihrer Arbeit in der Anästhesie oder auf der Intensivstation achten soll und welche Arbeiten sie nicht mehr übernehmen kann. Jederzeit steht er ihr als Berater und Ansprechpartner zur Verfügung. Denn verschiedene Arbeiten können für Mutter und Kind gefährlich sein: invasive Tätigkeiten, das betrifft Blutentnahmen, das Legen von Zugängen oder das Spritzen; Arbeiten mit Nothilfecharakter - sie können mental sehr belasten; Umgang mit kontaminierten Gegenständen oder Körperflüssigkeiten; Arbeiten in Räumen mit fruchtschädigenden Narkosegasen ( $\rightarrow$ Info Seite 124).

\section{Im Zweifel für die Gesundheit von Mutter und Kind}

Das Mutterschutzgesetz gibt nur einen Rahmen vor und ist nicht immer ganz eindeutig. Klar geregelt sind die Arbeitszeiten: nicht vor sechs Uhr morgens und nicht nach 20 Uhr abends. Klar ist auch das Verbot, regelmäßig mehr als fünf Kilogramm oder gelegentlich mehr als zehn Kilogramm zu heben oder zu tragen. Schwierig wird es aber bei der Frage, ob Schwangere in Schutzkleidung Kontakt mit MRSATrägern haben dürfen. Auch wenn ohnehin zehn Prozent der Bevölkerung zeitweise MRSA-Träger sind, ohne dass sie erkranken, und werdende Mütter eine höhere Immun-Kompetenz haben, gilt im Zweifelsfall immer: Der Schutz von Mutter und Kind hat Vorrang! Darauf achten auch die Kollegen. Das hat in der Kölner Uniklinik bisher gut geklappt: Von ihren acht Schwangeren war nur eine Mitarbeiterin im absoluten Beschäftigungsverbot, sechs Kolleginnen haben sich für eine Tätigkeit im Büro entschieden und eine hat die Arbeit in der Pflege mit nur einem Krankheitstag komplett - sogar ohne Urlaub vor der Mutterschutzfrist - durchgezogen.

\section{Straff organisiert}

Wenn das Baby geboren ist, plant Katja Mülder nach der achtwöchigen Babypause

noch drei Wochen Urlaub ein und möchte dann wieder im Job durchstarten. Sie bleibt aber realistisch: „So sieht mein Plan aus, aber niemand weiß im Vorfeld, ob das Kind so ,pflegeleicht' sein wird, dass alle Träume wahr werden.“

\section{VERGLEICH: KRANKENGELD UND BESCHÄFTIGUNGSVERBOT WÄHREND DER SCHWANGERSCHAFT}

Wird eine Mitarbeiterin während der Schwangerschaft vom Arzt länger krankgeschrieben, erhält sie aufgrund der Berufsunfähigkeit bis zu sechs Wochen Lohnfortzahlung vom Arbeitgeber. Danach zahlt ihr die Krankenkasse $\mathbf{7 0}$ Prozent ihres letzten vollen monatlichen Bruttolohns als Krankengeld bis zum Beginn der Mutterschutzfrist.

Erhält die Mitarbeiterin während ihrer Schwangerschaft vom Arzt hingegen ein Teil- oder absolutes Beschäftigungsverbot, muss der Arbeitgeber das Gehalt bis zum Beginn der Mutterschutzfrist in voller
Info Detaillierte Informationen gibt es in der Regel vom zuständigen Personalsachbearbeiter, vom Personalrat und von den regionalen Gewerbeaufsichtsämtern.

\section{GEFÄHRDUNGSBEURTEILUNG}

Höhe weiterzahlen. Die Höhe der Vergütung bemisst sich hier, genauso wie beim Krankengeld, am Durchschnittsverdienst der letzten drei Monate. Einmal- oder Sonderzahlungen wie zum Beispiel die Jahressonderzahlung werden dabei nicht berücksichtigt.

Viele Krankenhäuser beteiligen sich am Umlageverfahren (U2). Das heißt, sie bezahlen in die Umlagekasse ein wie in eine Versicherung und bekommen bei Eintreten eines Beschäftigungsverbots ihrer Mitarbeiterin die Lohnfortzahlung aus diesem Topf erstattet.

Arbeiten oder eine Arbeitsumgebung, die werdenden Müttern schaden können (Auszug aus der Verordnung zum Schutz der Mütter am Arbeitsplatz):

- Heben, Tragen oder Bewegen von Lasten ohne mechanische Hilfsmittel (regelmäßig mehr als fünf Kilogramm/ gelegentlich mehr als zehn Kilogramm)

- erhebliches Strecken, Beugen oder Hocken (beim Lagern von Patienten/beim Bettenmachen)

- Unfallgefahr (Ausrutschen auf nassen Böden/aggressive Patienten)

- Hitze, Kälte, Nässe (ständige Temperaturen unter 17 Grad Celsius)

- Erschütterungen oder Lärm > $80 \mathrm{~dB}$ oder Lärmspitzen

- andere schädliche physikalische Einwirkungen (Magnetfelder von Kernspintomografen)

- Fahrtätigkeiten oder die Begleitung

- Arbeiten im Stehen mehr als vier Stunden/Tag (nach Ablauf des fünften Schwangerschaftsmonats)

- Bedienen von Geräten mit hoher Fußbeanspruchung

- Arbeiten mit Gefahrenstoffen (wie Blei, Quecksilber, Mitosehemmstoffe in Zytostatika, in die Haut eintretende Stoffe, krebserzeugende, erbgutverändernde, fruchtschädigende, fortpflan- zungsgefährdende Stoffe in Halothan, Grenzwertüberschreitung bei schädlichen Stoffen wie Narkosegasen oder Desinfektionsmitteln)

- Umgang mit biologischen Arbeitsstoffen (erhöhtes Infektionsrisiko z. B. mit Toxoplasma, Rötelnvirus, Hepatitisviren, Zytomegalie, Ringelröteln etc.) ohne ausreichenden Schutz oder Nachweis einer Immunisierung

- ungeschützter Umgang mit potenziell infektiösem Material (wie Blut, Blutbestandteile, sonstigen Körperflüssigkeiten) oder Gefahr der Verletzung mit stechenden, schneidenden, rotierenden, zerbrechlichen Geräten und Instrumenten

- ionisierende Strahlung/nicht ionisierende Strahlung/radioaktive Stoffe

- vorgeschriebenes Arbeitstempo

- Nachtarbeit

- Mehrarbeit

- Sonntagsarbeit

Die Arbeitsbedingungen und gegebenenfalls die Arbeitszeiten sind so umzugestalten, dass eine Gefährdung ausgeschlossen ist. 


\section{GEFAHRENQUELLE NARKOSEGASE}

Narkosegase oder Inhalationsnarkotika zählen zu den Gefahrstoffen. Neben dem Lachgas unterscheidet man zwischen halogenierten Kohlenwasserstoffen (Halothan) und Ethern (Desfluran, Enfluran, Isofluran, Sevofluran). In Räumen, in denen mit Narkosemitteln gearbeitet wird, können werdende oder stillende Mütter schädlichen Einwirkungen von gesundheitsgefährdenden Gasen und Dämpfen im Sinne von $\S 4$ Abs. 1 und 2 Nr. 6 und $\S 6$ Abs. 3 MuSchG ausgesetzt sein. Eine Beschäftigung dort ist nur dann zulässig, wenn der Luftgrenzwert für diese Gefahrstoffe sicher und dauerhaft unterschritten wird $(\rightarrow$ Tab. 1). Sofern es sich um Intubationsnarkosen (geschlossene Verfahren) handelt, kann diese Bedingung erfüllt werden. Dies muss durch ausreichend häufige Messungen nachgewiesen werden. Dies gilt aber nicht für Maskennarkosen, die besonders bei Kindern angewendet werden. Hierbei kann es zu einer Überschreitung der Luftgrenzwerte kommen. Eine abschließende Bewertung ist gegenwärtig nicht möglich.

- Lachgas (Distickstoffmonoxid- $\mathrm{N}_{2} \mathrm{O}$ ) hat den Grenzwert $180 \mathrm{mg} / \mathrm{m}^{3}$ bzw. $100 \mathrm{ml} /$ $\mathrm{m}^{3}$ (Technische Regel für Gefahrstoffe TRGS 900, Stand 10/2000). Nach Erkenntnissen der DFG-Kommission ist eine abschließende Beurteilung nicht möglich. Daher wird aus präventivmedizinischen Gründen eine Beschäftigung von werdenden Müttern auch bei Einhaltung des Grenzwertes derzeit nicht emp- fohlen. Unter allgemeinen arbeitsmedizinischen Aspekten wird Xenon (XE) als Alternative zu Lachgas positiv beurteilt. Die relativ hohen Kosten stehen derzeit der weiteren Verbreitung entgegen.

- Halothan (2-Brom-2-chlor-1,1,1-trifluorethan) hat den Grenzwert $41 \mathrm{mg} / \mathrm{m}^{3}$ bzw. $5 \mathrm{ml} / \mathrm{m}^{3}$. Halothan findet sich in die Gruppe $B^{*}$ : Das Risiko einer Fruchtschädigung muss als wahrscheinlich unterstellt werden. Es besteht auch bei Einhaltung der Grenzwerte eine Gefährdung.

- Enfluran (2-Chlor-1,1,2-trifluorethyldifluormethylether) hat den Grenzwert $20 \mathrm{ml} / \mathrm{m}^{3}$. Enfluran findet sich in Gruppe $C^{*}$ : Das Risiko einer Fruchtschädigung muss bei Einhaltung der Grenzwerte nicht befürchtet werden.

- Isofluran (1-Chlor-2,2,2-trifluorethyldifluormethylether) gehört zur Gruppe der häufig verwendeten fluorierten Narkosemittel. Zurzeit gibt es nur einen Vorschlag für einen Grenzwert von $80 \mathrm{mg} / \mathrm{m}^{3}$ (TRGS 900).

Für die anderen genannten Inhalationsnarkotika bestehen gegenwärtig weder Grenzwerte noch wissenschaftlich gesicherte Aussagen über eine mögliche Fruchtschädigung bei Schwangerschaft. Die arzneimittelrechtliche Überprüfung hat ergeben, dass Sevofluran und Desfluran eine geringere Toxizität aufweisen als bisher übliche Anästhesiemittel. Keine Erkenntnisse liegen jedoch hinsichtlich der reproduktionstoxischen Effekte vor.
Die Beschäftigung kann in Operationsbereichen ohne geeignete Atemluftrückführung und Absaugung, in denen die Atemluft durch erhöhte Konzentrationen von Narkosegasen belastet wird, untersagt werden.

Für die festzulegenden Schutzmaßnahmen gibt es arbeitsmedizinisch-toxikologische Empfehlungen des Berufsgenossenschaftlichen Instituts für Arbeitsschutz (BIA) zur Überwachung von Arbeitsbereichen für Anästhesiearbeitsplätze in Operationssälen und Aufwachräumen. Bei Anwendung der Empfehlungen kann von einer dauerhaft sicheren Einhaltung der Grenzwerte ausgegangen werden. Grundvoraussetzung für die Beschäftigung einer Schwangeren beim Einsatz der genannten Narkosemittel im OP-Raum ist daher die umfassende Beachtung aller BIA-Empfehlungen (Berufsgenossenschaftliches Institut für Arbeitsschutz Anästhesiearbeitsplätze Operationssäle [1017]), insbesondere:

- Einhaltung der Arbeitsschutzanforderungen der TRGS 525 (Umgang mit Gefahrstoffen in Einrichtungen zur humanmedizinischen Versorgung, Kapitel 6),

- Beschränkung auf die in der BIA-Empfehlung 1017 beschriebenen Narkoseverfahren, bei denen der Bewertungsindex für Anästhesiegase eingehalten wird.

Quelle: Regierungspräsidien Baden-Württemberg, Fachgruppe Mutterschutz

\section{TABELLE 1: LUFTGRENZWERTE FÜR GEFAHRSTOFFE}

\begin{tabular}{|c|c|c|c|c|c|c|}
\hline Gefahrenstoff & \multicolumn{2}{|c|}{$\begin{array}{l}\text { MAK (AGW) } \\
\text { (Maximale Arbeitsplatzkonzentration) } \\
\text { (TRGS 900) }\end{array}$} & $\begin{array}{l}\text { Spitzen- } \\
\text { begrenzung }\end{array}$ & \multicolumn{2}{|c|}{ Schwangerschaftsgruppe } & $\begin{array}{l}\text { BAT (BGW) } \\
\text { (Biologischer } \\
\text { Arbeitsplatztoleranzwert) }\end{array}$ \\
\hline $\begin{array}{l}\text { Distickstoffmonoxid } \\
\text { (Lachgas) }\end{array}$ & 180 & 100 & 2 & $C^{*}$ & - & - \\
\hline Halothan & 41 & 5 & 8 & $\mathrm{~B}^{*}$ & $\mathrm{RE} 2 * *$ & Triflouressigsäure $2,5 \mathrm{mg} / \mathrm{l}$ Blut \\
\hline Isofluran & \multicolumn{2}{|l|}{ II b* } & - & \multicolumn{3}{|l|}{-} \\
\hline Sevofluran & \multicolumn{2}{|l|}{-} & - & \multicolumn{3}{|l|}{-} \\
\hline Desfluran & \multicolumn{2}{|l|}{-} & - & \multicolumn{3}{|l|}{-} \\
\hline Xenon & \multicolumn{2}{|l|}{-} & - & \multicolumn{3}{|l|}{-} \\
\hline
\end{tabular}

* MAK- und BAT-Wert-Liste Mitteilung 44 der Senatskommission der DFG 2008:

Gruppe B: Ein Risiko der Fruchtschädigung muss als wahrscheinlich unterstellt werden

Gruppe C: Ein Risiko der Fruchtschädigung braucht bei Einhaltung des MAK-Wertes nicht befürchtet werden.

Gruppe II b: Stoffe für die derzeit keine MAK-Werte aufgestellt werden können.

** TRGS 905: RE2: Fruchtschädigend, Kategorie 2 


\section{INDIVIDUELLE BESCHÄFTIGUNGSVERBOTE}

Das Mutterschutzgesetz und die Verordnung zum Schutze der Mütter am Arbeitsplatz stecken einen Sicherheitsrahmen, der sich am normalen Schwangerschaftsverlauf orientiert. Jedoch können individuelle konstitutionelle Bedingungen der Schwangeren, individuelle Beschwerden und gesundheitliche Beeinträchtigungen einzelner Frauen darin nicht ausreichend berücksichtigt werden. Der Gesetzgeber hat deshalb in $\S 3$ des Mutterschutzgesetzes ein individuelles Beschäftigungsverbot verankert, das vom behandelnden Arzt festgelegt werden kann. In §3 Abs. 1 Mutterschutzgesetz heißt es dazu:

„Werdende Mütter dürfen nicht beschäftigt werden, soweit nach ärztlichem Zeugnis Leben oder Gesundheit von Mutter oder Kind bei Fortdauer der Beschäftigung gefährdet ist.“ Diese Regelung bietet dem Arzt die Möglichkeit zu bestimmen, welche Tätigkeit im Hinblick auf die individuellen körperlichen Gegebenheiten der werdenden Mutter beziehungsweise des ungeborenen Kindes eine solche Gefahr darstellen können und deshalb nicht mehr ausgeübt werden dürfen. Der Entscheidungsspielraum des Arztes erstreckt sich von Beschränkungen hinsichtlich Art, Umfang und Dauer bestimmter Tätigkeiten bis hin zum Verbot jeglicher Tätigkeit.
Von der Regelung werden neben den normalen Beschwerden der Schwangerschaft sowie typischen Symptomen für eine Gefährdung der Schwangerschaft auch pathologische Symptome wie zum Beispiel Erbrechen, schwangerschaftsbedingte Kreislauflabilität, Anämie, Risiko einer Frühgeburt, Thromboseneigung, aber auch psychische Belastungen durch eine Beschäftigung, die sich nachteilig auf den Verlauf der Schwangerschaft auswirken können, erfasst. Die Arbeit, die nach ärztlichem Zeugnis nicht oder nur in beschränktem Umfang von der Schwangeren ausgeübt werden darf, kann zwar im Allgemeinen als ungefährlich eingeschätzt werden, für die Schwangere aufgrund subjektiver Gegebenheiten jedoch zu Beschwerden führen, die ihre Gesundheit oder die des Kindes gefährden können. Dies kann schon bei Essensgerüchen der Fall sein. Das ärztliche Beschäftigungsverbot wird durch Vorlage eines schriftlichen Zeugnisses eines approbierten Arztes beim Arbeitgeber wirksam. Das Attest ist klar abzufassen. Es muss die Rechtsgrundlage (§3 Mutterschutzgesetz), die voraussichtliche Geltungsdauer, Umfang und Art der untersagten Tätigkeit sowie die Art der Gefährdung möglichst genau und allgemein verständlich darstellen. Es ist auch möglich darzustellen, welche Art von
Tätigkeit die Schwangere ausüben darf. Auch dem medizinisch nicht vorgebildeten Arbeitgeber muss es möglich sein, Umfang und Dauer des Beschäftigungsverbots zu erkennen. Das ärztliche Beschäftigungsverbot ist sowohl für den Arbeitgeber als auch für die Arbeitnehmerin bindend. Die Kosten der Bescheinigung trägt die Arbeitnehmerin. Bei Zweifeln an der Richtigkeit des Attests kann der Arbeitgeber - unter Beachtung des Rechts der Schwangeren auf freie Arztwahl - eine Nachuntersuchung durch einen anderen Arzt verlangen. Die Kosten hierfür hat der Arbeitgeber zu tragen. Bis zur Vorlage des Ergebnisses der Nachuntersuchung darf die Arbeitnehmerin nur entsprechend dem ursprünglichen Attest beschäftigt werden. Während der Zeit, in der nach ärztlichem Zeugnis eine Beschäftigung der Frau ganz oder teilweise untersagt ist, hat die Frau ebenso wie bei den gesetzlich normierten Beschäftigungsverboten Anspruch auf den Durchschnittsverdienst entsprechend \$11 Mutterschutzgesetz.

Quelle: Regierungspräsidien Baden-Württemberg, Fachgruppe Mutterschutz

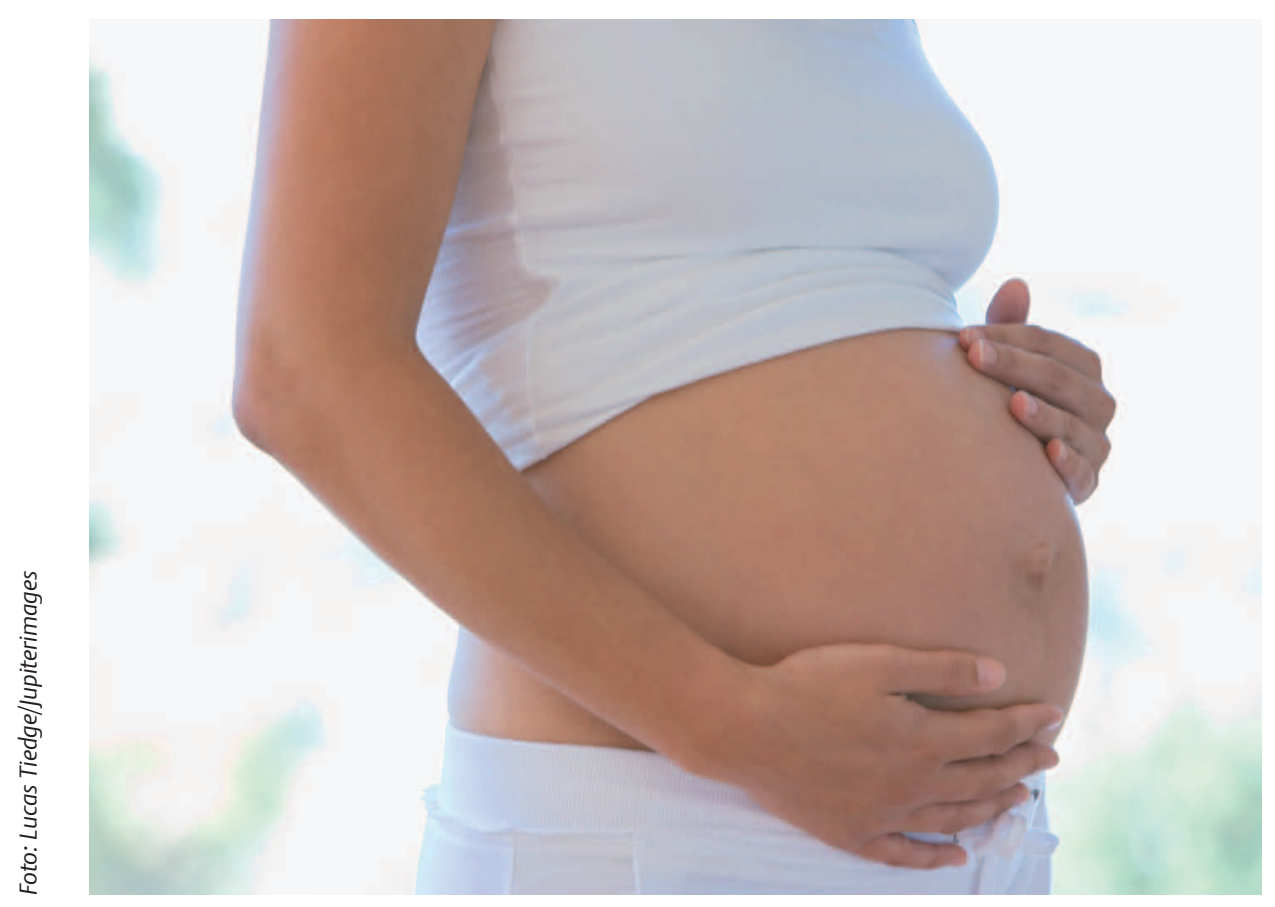

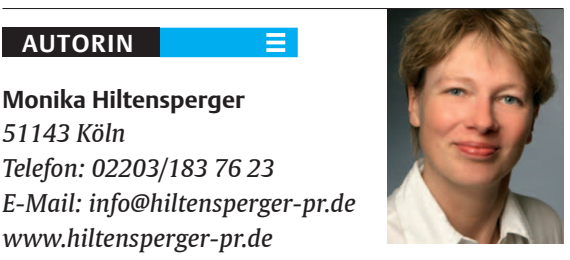

BIBLIOGRAFIE

DOI 10.1055/s-0032-1310329

Intensiv 2012; 20 (3): 121-125

(c) Georg Thieme Verlag KG

Stuttgart · New York · ISSN 0942-6035 